\title{
riccafd
}

Revista Iberoamericana de Ciencias de la Actividad Física y el Deporte

\section{EFECTOS DEL PIAFARC SOBRE LA CONDICIÓN FÍSICA Y LA DENSITOMETRÍA EN OBESOS}

PIAFARC EFFECTS ON PHYSICAL CONDITION AND DENSITOMETRY IN OBESE

\section{Monleón C. ${ }^{1}$, Fargueta, M. ${ }^{2}$ y Elvira, L. ${ }^{3}$}

1. Doctora Cristina Monleón García; Universidad Católica de Valencia "San Vicente Mártir"; España; cristina.monleon@ucv.es.

2. Profesora María Fargueta Espert; Universidad Católica de Valencia "San Vicente Mártir"; España; maria.fargueta@ucv.es.

3. Doctora Laura Elvira Macagno; Universidad Católica de Valencia "San Vicente Mártir"; España; laura.elvira@ucv.es.

El presente trabajo se ha realizado gracias a la financiación de una Beca Predoctoral para la Contratación de Personal Investigador en Formación de la primera autora en la Universidad Católica de Valencia.

Código UNESCO: 3212 Salud Pública

Clasificación Consejo de Europa: 17 Actividad Física y Salud

Recibido el 3 de julio de 2015

Aceptado el 6 de octubre de 2015

Correspondencia:

Cristina Monleón García: cristina.monleon@ucv.es

\section{RESUMEN}

La obesidad es una enfermedad sistémica, crónica y multicausal que afecta a todas las edades, sexos y condiciones sociales. Las alteraciones a nivel músculo-esquelético son evidentes, repercutiendo en estructuras óseas, articulares y desencadenando enfermedades que conllevan un incremento destacado en el gasto sanitario. El objetivo del estudio fue comparar el efecto de dos programas de actividad física basado en actividades rítmicas con control nutricional (PIAFARC). El programa de actividad física tuvo una duración de 8 meses para cada uno de los dos estudios y se aplicó a dos muestras de 34 adultos obesos. Se midieron variables de condición física y densitometría. Los resultados de la comparativa muestran diferencias significativas para el equilibrio $(p=0,018)$ y la fuerza en piernas $(p=0,045)$ a favor del PIAFARC1 y PIAFARC2 respectivamente. 
PALABRAS CLAVE: Obesidad; condición física; densitometría ósea; baile; calidad de vida.

\section{ABSTRACT}

Obesity is a multi-causal systemic disease, that affects all ages, sexes and social conditions. Alterations to muscle-skeleton level are evident, affecting to bone structures, joins and triggering diseases involving a prominent increase in healthcare spending. The study aimed was to compare the effect of two physical activity programs based on rhythmic activities with nutritional management (PIAFARC). The physical activity program lasted eight months for each of the two studies and applied to two samples of 34 obese adults. Fitness variables and densitometry were measured. The comparative results show significant differences to the balance $(p=0.018)$ and strength in legs $(p=$ 0.045 ) in favour of PIAFARC 1 and PIAFARC2 respectively.

KEYWORDS: Obesity, physical condition, bone densitometry; dance; quality of life.

\section{INTRODUCCIÓN}

La obesidad es una enfermedad sistémica, crónica y multicausal (1) considerada en la actualidad como uno de los problemas más frecuentes en la sociedad, afectando principalmente a las sociedades desarrolladas, y abarcando todas las edades, sexos y condiciones sociales (2). Podríamos referirnos a la obesidad como la enfermedad del siglo XXI a causa de su elevada prevalencia en la sociedad, repercutiendo en un aumento considerable de los costes en la atención sanitaria (3). La repercusión económica a nivel sanitario viene determinada por la variedad de enfermedades crónicas asociadas a dicha patología (4), como pueden ser entre otras, las enfermedades cardiovasculares, destacando la cardiopatía isquémica y cerebrovascular (5), enfermedades de carácter pulmonar, diabetes, dislipidemias e hipertensión arterial (6).

La obesidad, así como el exceso de peso estarán vinculadas directamente con alteraciones a nivel músculo-esquelético, repercutiendo en las estructuras óseas y articulares, debido al exceso de fuerza que deben soportar y provocando finalmente una alteración de la mecánica corporal $(7,8)$. Asumimos también, que es el factor desencadenante de enfermedades como artritis, deformidades óseas y lesiones articulares (5). En el caso de personas de mediana edad y personas mayores, puede actuar como predictor de una limitación hacia la movilidad (9). Además, estas alteraciones harán que se observen bajos niveles de condición física, tanto en la aptitud 
cardiorrespiratoria, fuerza, resistencia, flexibilidad de columna, así como también se podrá ver reducido el rango de movimiento articular (10). Los niveles bajos de condición física tienen sin duda repercusión en las tareas del día a día, dificultando a las personas llevar a cabo acciones tan comunes como subir escaleras o hacer actividades cotidianas durante un tiempo prolongado (11).

Una manera de intervenir o incluso prevenir alteraciones musculo esqueléticas en personas obesas sería mediante la modificación del estilo de vida, siguiendo un correcto plan de alimentación combinado con la realización de actividad física constante y regular (5). En esta línea Wearing et al. (12) afirman que la actividad física reduce el riesgo de padecer osteoartritis, además de reducir también el riesgo de desórdenes en el disco espinal, el dolor de espalda, así como también de posibles trastornos en los tejidos blandos. Además, la práctica de ejercicio físico mejorará capacidades físicas como la fuerza, la resistencia y la flexibilidad (13)

Asumimos entonces, que la actividad física tiene un valor preventivo y se recomienda como el primer paso en la prevención de la osteoporosis, así como, el aumento de la fuerza muscular y con ello la reducción de las caídas y fracturas (14), mejorando por lo tanto la calidad de vida de las personas obesas, no solo desde un punto de vista físico o fisiológico sino también psicosocial, ya que la práctica de actividad física de manera regular aumenta la capacidad de tomar decisiones y la confianza en sí mismo (15).

El ejercicio más adecuado sería un trabajo de fuerza resistencia (16). En este caso se presenta un programa de actividad física mediante el baile, siendo este un método que facilita tanto la motivación como la interacción social (17).

Además los programas basados en bailes o danzas tienen efectos sobre el equilibrio, la fuerza, la coordinación, el ritmo, la agilidad y la resistencia entre otros. Estos efectos se deben a la presencia de cierta dificultad al ejecutar los movimientos, a los cambios de dirección que se realizan y todo ello a diferentes intensidades y siguiendo el ritmo de la música (17-19).

El objetivo del estudio fue analizar el efecto de dos programas de actividad física basados en actividades rítmicas y coreográficas con control nutricional en dos poblaciones obesas sobre las variables de condición física y densitometría ósea. 


\section{MATERIAL Y MÉTODOS}

\section{Participantes}

La selección de la muestra fue mediante muestreo no probabilístico consecutivo a partir de las personas interesadas en la participación en el estudio del departamento de Endocrinología y Nutrición de un centro de salud.

Los sujetos que estaban interesados en participar firmaban un consentimiento voluntario e informado de participación en el estudio y además cumplimentaban un cuestionario de hábitos de Actividad Física y Salud para disponer de una anamnesis completa, la cual era utilizada a posteriori para determinar si cumplían o no los criterios de inclusión.

La muestra total del primer estudio estuvo formada 39 participantes, de los cuales 34 completaron todas las sesiones (25 mujeres y 9 hombres), con edades $50,43 \pm 10,57$ y $38,37 \pm 4,82$ IMC.

La muestra del segundo estudio que se llevó a cabo, estuvo formado por 34 participantes (27 mujeres y 7 hombres) con una edad media de 48,724 $\pm 12,34$ años y un IMC de 38,55 \pm 6,23.

Para ambos estudios se tenía en cuenta que fueran participantes sedentarios, que tuviesen un IMC $\geq 30 \mathrm{Kg} / \mathrm{m} 2$, que firmasen el consentimiento informado y voluntario, y que pudiesen someterse a un programa de entrenamiento después de ser valorados a nivel médico.

\section{Programa de ejercicio}

Se diseñó y aplicó un Programa de Intervención con Actividades Rítmicas y Coreográficas (PIAFARC) en dos programas diferentes con una duración cada uno de ellos de 8 meses.

EI PIAFARC para el primer año (PIAFARC1) tuvo una duración de 8 meses y se realizaban 3 sesiones semanales, dos de ellas presenciales de 60 minutos de duración (10 minutos de calentamiento, 40 minutos de parte principal y 10 minutos de vuelta a la calma) en la que se trabajaba mediante actividades rítmicas y coreográficas así como juegos rítmicos, y la tercera sesión era de tipo tónico mediante el uso de bandas elásticas, y era realizada sin supervisión. No obstante, el primer día de programa se les instruía en la realización de los ejercicios que se les proporcionaba mediante un dossier. Tras cada sesión presencial, mediante la escala de percepción del esfuerzo de Borg se valoraba 
la intensidad media al finalizar cada sesión (20) con el objetivo de controlar una intensidad moderada. Asimismo, los participantes de este estudio llevaban un control y seguimiento nutricional por la Unidad de Endocrinología y Nutrición de un Centro de Salud. El porcentaje de asistencia al programa fue del $67,07 \% \pm$ 16,45 .

EI PIAFARC para el segundo año (PIAFARC2) también tuvo 8 meses de duración, pero en este caso el programa consistía en 2 sesiones presenciales con la misma duración y fases que el primer programa. La tercera sesión fue excluida debido a la dificultad de controlar su realización. La intensidad moderada que se buscaba con las sesiones también fue controlada mediante la escala de percepción del esfuerzo de Borg. En este caso los participantes llevaban un control y seguimiento nutricional exhaustivo por parte del propio equipo investigador y se aplicaron talleres nutricionales como apoyo al tratamiento de la dieta. El porcentaje de asistencia al programa en este segundo estudio fu del $66,71 \% \pm 20,08$.

El diseño del estudio cumplió con los requisitos recogidos en la declaración de Helsinki de 1975.

\section{Materiales y procedimiento de los tests}

Las variables objeto de estudio fueron la condición física y la densitometría ósea. Respecto a las relacionadas con la condición física se midió la flexibilidad de espalda baja e isquiotibiales en ambas piernas mediante el test de Sit-and Reach $(21,22)$, la capacidad aeróbica mediante el test de 6 minutos permitiendo no sólo andar sino también correr o combinar ambos casos (23), el equilibrio dinámico hacia detrás mediante el Tandem Walk Backward Test (24), evaluándolo no sólo con el control del tiempo que tardan en recorrer una línea de 6 metros, sino mediante la penalización de las salidas de la línea y las veces que no realiza la posición de tándem con los pies; la flexibilidad de los miembros superiores mediante el test de Flexibilidad de Cintura Escapular para la medición de la capacidad de amplitud articular (25), la fuerza de las extremidades superiores e inferiores mediante una barra de $3 \mathrm{Kg}$ enganchada al dinamómetro isoinercial T-Force V. 2.35 (Ergotech, Murcia, España). En cuanto a variables de densitometría ósea, para su valoración a nivel de calcáneo se utilizó el Densitómetro Óseo SONOST-3000 (OsteoSys Co., Ltd, Seoul, Korea). El SONOST-3000 calcula la densidad mineral ósea a nivel de calcáneo así como da información sobre el riesgo de fractura por la Atenuación de ultrasonido por banda ancha (BUA), el Índice de Calidad del Hueso (BQI), T-score y Z-score. El uso de este tipo de equipo es debido a que el calcáneo es un lugar adecuada de medición debido a su alto contenido trabecular y 
accesibilidad (26) así como un lugar donde se puede apreciar el efecto de la carga del peso en la actividad física (27).

\section{Análisis estadístico}

Para la comparación de los resultados obtenidos para cada estudio, se extrajeron puntuaciones de ganancia para cada variable tras la intervención con el fin de obtener la mejora obtenida. Los descriptivos se muestran con medias y desviación típica.

Para conocer si habían diferencias significativas entre los resultados de los dos programas se realizaron pruebas $t$ para muestras independientes. El nivel de significación considerado fue de $p<0,05$.

Para el análisis estadístico se utilizó el software estadístico SPSS versión 20 (IBM, Somers, NY, USA)

\section{RESULTADOS}

Los resultados obtenidos de la comparativa entre el primer y segundo estudio en cuanto a las variables de densitometría ósea están detalladas en la Tabla 1.

Tabla 1. Descriptivos, prueba de normalidad y comparativa de grupos para las puntuaciones de ganancia de las variables de densitometría ósea.

\begin{tabular}{llccccc}
\hline \multicolumn{2}{l}{ Variable/Grupo } & $\mathrm{n}$ & Media & $\mathrm{DE}$ & $\begin{array}{c}\text { S-W Sig. } \\
\text { (bilateral) }\end{array}$ & $\mathrm{t}$ (p-valor) \\
\hline \multirow{2}{*}{ BUA } & PIAFARC1 & 34 & 1,086 & 0,298 & $-0,1509(\mathrm{p}=0,131)$ & 9,426 \\
& PIAFARC2 & 34 & 6,824 & 0,000 & & 20,001 \\
\multirow{2}{*}{ BQI } & PIAFARC1 & 34 & 3,195 & 0,603 & $-1,447(p=0,148)$ & 7,966 \\
& PIAFARC2 & 34 & 1,417 & 0,004 & & 15,501 \\
\multirow{2}{*}{ T-score } & PIAFARC1 & 34 & 0,172 & 0,608 & $0,622(p=0,537)$ & 0,428 \\
& PIAFARC2 & 34 & 0,072 & 0,003 & & 0,834 \\
\multirow{2}{*}{ Z-score } & PIAFARC1 & 34 & 0,201 & 0,637 & $-0,957(p=0,339)$ & 0,465 \\
& PIAFARC2 & 34 & 0,137 & 0,074 & & 0,866 \\
\hline
\end{tabular}

Nota: $D E$, Desviación estándar; S-W Sig. (bilateral), significancia por ShapiroWilk. ${ }^{*} p<0,05 ;{ }^{* *} p<0,01 ;{ }^{* * *} p<0,001$

Los resultados obtenidos no muestran diferencias significativas tras la comparativa de ambos estudios en ninguna de las variables estudiadas.

Respecto a la comparativa de las variables de la condición física se detallan en la Tabla 2.

16 EFECTOS DEL PIAFARC SOBRE LA CONDICIÓN FÍSICA Y LA DENSITOMETRÍA EN OBESOS 
Tabla 2. Descriptivos, prueba de normalidad y comparativa de grupos para las puntuaciones de ganancia de las variables de condición física.

\begin{tabular}{|c|c|c|c|c|c|c|}
\hline \multicolumn{2}{|c|}{ Variable/Grupo } & \multirow{2}{*}{$\frac{n}{32}$} & \multirow{2}{*}{$\frac{\text { Media }}{48,438}$} & \multirow{2}{*}{$\frac{\mathrm{DE}}{0,992}$} & \multirow{2}{*}{$\begin{array}{c}\begin{array}{c}\text { S-W Sig. } \\
\text { (bilateral) }\end{array} \\
0,892(p=0,376)\end{array}$} & \multirow{2}{*}{$\frac{t(p \text {-valor })}{114,494}$} \\
\hline$R$ & PIAFARC1 & & & & & \\
\hline Resistencle & PIAFARC2 & 31 & 26,807 & 0,053 & \multirow{3}{*}{$-2,434(p=0,018)$} & 74,357 \\
\hline \multirow{2}{*}{ Equilibrio } & PIAFARC1 & 34 & $-10,992$ & 0,819 & & 11,308 \\
\hline & PIAFARC2 & 32 & $-5,103$ & 0,121 & & 7,944 \\
\hline \multirow{2}{*}{$\begin{array}{l}\text { FI. pierna } \\
\text { dchab }^{b}\end{array}$} & PIAFARC1 & 34 & 2,897 & 0,711 & \multirow[t]{2}{*}{$-1,561(p=0,124)$} & 7,421 \\
\hline & PIAFARC2 & 32 & 5,378 & 0,111 & & 5,234 \\
\hline FI. pierna & PIAFARC1 & 34 & 3,441 & 0,588 & \multirow[t]{2}{*}{$-1,469(p=0,147)$} & 6,019 \\
\hline$i z q^{b}$ & PIAFARC2 & 32 & 0,45 & 0,414 & & 5,01 \\
\hline Fuerza & PIAFARC1 & 31 & 0,278 & 0,532 & \multirow[t]{2}{*}{$-0,454(p=0,650)$} & 0,354 \\
\hline brazos $^{\mathrm{c}}$ & PIAFARC2 & 30 & 0,449 & 0,000 & & 1,306 \\
\hline \multirow{2}{*}{$\begin{array}{l}\text { Fuerza } \\
\text { piernas }^{c}\end{array}$} & PIAFARC1 & 32 & 0,121 & 0,109 & \multirow[t]{2}{*}{$-2,051(p=0,045)$} & 0,206 \\
\hline & PIAFARC2 & 31 & 0,226 & 0,404 & & 0,202 \\
\hline
\end{tabular}

Nota: $D E$, Desviación estándar; S-W Sig. (bilateral), significancia por ShapiroWilk; Fl, Flexibilidad; dcha, Derecha; izq, Izquierda. La unidad para cada variable es: ${ }^{a}$ metros; ${ }^{b} \mathrm{c} / \mathrm{m} ;{ }^{c} \mathrm{~m} / \mathrm{s}$. ${ }^{*} \mathrm{p}<0,05 ;{ }^{* *} \mathrm{p}<0,01 ;{ }^{* * *} \mathrm{p}<0,001$

Los resultados obtenidos de la comparativa nos muestran diferencias significativas en la variable de equilibrio $t(64)=-2,434, p=, 018$; TE=0,29; siendo los resultados obtenidos mayores para el PIAFARC1 respecto al PIAFARC2. Los resultados obtenidos de la fuerza de miembros inferiores $t(61)$ $=-2,051, p=, 045, \mathrm{TE}=0,25$, muestran una mayor mejora en el segundo estudio respecto al primero.

\section{DISCUSIÓN}

Intervenciones con ejercicios programados se han destacado como herramienta útil y una estrategia eficaz para la ayuda en poblaciones obesas (28). En este sentido, diferentes autores han empleado las actividades rítmicas y bailes como método en sus programas en diferentes poblaciones, obteniendo resultados positivos no sólo a nivel de condición física, sino también en variables antropométricas (29-33).

Los resultados obtenidos respecto a la densitometría ósea, no muestran diferencias significativas. Sin embargo se puede observar el beneficio de las 
variables relacionadas tras los 2 PIAFARC que podría estar dado por la zona de medición (calcáneo) por ser una zona específica de soporte de peso y expuesto a carga mecánica (34).

Respecto a los resultados obtenidos en la condición física sólo se obtuvieron diferencias en el equilibrio y en la fuerza de miembros inferiores. Respecto al equilibrio, se obtuvo una mayor mejora en el primer estudio respecto al segundo. Esto podría ser debido a que en el primer estudio se empezaba con una peor condición física respecto al segundo estudio, por lo que el rango de mejora es mayor. También podría deberse al trabajo realizado con gomas de forma autónoma. Pese a ello, esta mejora del equilibrio tanto en el PIAFARC1 como en el PIAFARC2 sugieren una reducción del riesgo de caídas así como una prevención del dolor de espalda (35). En cuanto a la fuerza de miembros inferiores fue mayor la diferencia en el segundo estudio. En este caso, pensamos que podría ser debido a que en este segundo estudio se produce una mayor pérdida de peso, producido por el mayor control nutricional y la implementación de talleres nutricionales.

Los datos obtenidos son muy relevantes debido a que tanto el equilibrio como la fuerza en miembros inferiores son fundamentales en la locomoción y en las actividades de la vida diaria.

\section{CONCLUSIONES}

Los resultados obtenidos de la comparativa entre los dos PIAFARC, nos llevan a concluir que los programas de actividad física basados en actividades rítmicas y coreográficas permiten una mejora de la condición física en personas obesas, si bien, el introducir sesiones de trabajo en casa puede favorecer un mejor control del cuerpo, obteniendo mejoras mayores en el equilibrio.

Por otro lado, el tener un control nutricional exhaustivo permitirá mayores mejoras en el IMC, lo que favorecerá el mantenimiento o la mejora de la funcionalidad y movilidad de personas con obesidad. 


\section{REFERENCIAS}

1. Barrera Cruz A, Rodríguez González A, Molina Ayala MA. Escenario actual de la obesidad en México. Rev Med Inst Mex Seguro Soc. 2013;51(3):292-9.

2. Bauzá, C. Obesidad, causas, consecuencias y tratamiento. Madrid: El Emporio Libros; 2005.

3. Monleón C, Pablos A, Carnide F, Martín M, Pablos C. Effects of a rhythmic and choreographic program in obese and overweight participants. Nutr Hosp [Internet]. 2014 [cited 2014 Sep 8];30(3). Available from: http://www.aulamedica.es/gdcr/index.php/nh/article/view/7365

4. Sánchez L, Sánchez C, Alfonso A. Valoración de un programa de ejercicio físico estructurado en pacientes con obesidad mórbida pendientes de cirugía bariátrica. Nutr Hosp. 2014; 29(1): 64-72.

5. Rubio MA, Salas-Salvadó J, Barbany M, Moreno B, Aranceta J, Bellido D, et al. Consenso SEEDO 2007 para la evaluación del sobrepeso y la obesidad y el establecimiento de criterios de intervención terapéutica. Rev Esp Obes. 2007;5(3):135-75.

6. Chavarría S. Definición y criterios de obesidad. Nutr Clínica. 2002;5(4):236-40.

7. Capodaglio P, Castelnuovo G, Brunani A, Vismara L, Villa V, Capodaglio EM. Functional limitations and occupational issues in obesity: a review. Int J Occup Saf Ergon. 2010;16(4):507-23.

8. Ranavolo A, Donini LM, Mari S, Serrao M, Silvetti A, lavicoli S, et al. LowerLimb joint coordination pattern in obese subjects. BioMed Res Int. 2013;1-9.

9. Houston DK, Ding J, Nicklas BJ, Harris TB, Lee JS, Nevitt MC, et al. Overweight and Obesity Over the Adult Life Course and Incident Mobility Limitation in Older Adults: The Health, Aging and Body Composition Study. Am J Epidemiol. 2009;169(8):927-36.

10. Miller CT, Fraser SF, Levinger I, Straznicky NE, Dixon JB, Reynolds J, et al. The Effects of Exercise Training in Addition to Energy Restriction on Functional Capacities and Body Composition in Obese Adults during Weight Loss: A Systematic Review. PLoS ONE. 2013;8(11):e81692.

11. Browning RC. Locomotion Mechanics in Obese Adults and Children. Curr Obes Rep. 2012;1(3):152-9.

12. Wearing S, Henning E, Byrne N, Steele J, Hills A. Musculoskeletal disorders associated with obesity: a biomechanical perspective. Obes Rev. 2006;7(3):239-50.

13. De Souza Santos CA, Dantas EEM, Moreira MHR. Correlation of physical aptitude; functional capacity, corporal balance and quality of life (QoL) among elderly women submitted to a post-menopausal physical activities program. Arch Gerontol Geriatr. 2011;53(3):344-9.

14. Kai MC, Anderson M, Lau E. Exercise interventions: defusing the world's osteoporosis time bomb. Bull World Health Organ. 2003;81(11):827-30.

15. Delgado $M$. Efectos de un programa de tratamiento multidisciplinar en obesos mórbidos y obesos con comorbilidades cyidatos a cirugía bariaátrica. Nutr Hosp. 2015;31(05). Available from: http://www.aulamedica.es/gdcr/index.php/nh/article/view/8569

16. Hills AP, Hennig EM, Byrne NM, Steele JR. The biomechanics of adipositystructural and functional limitations of obesity and implications for movement. Obes Rev. 2002;3(1):35-43.

17. Granacher U, Muehlbauer T, Bridenbaugh SA, Wolf M, Roth R, Gschwind $Y$, et al. Effects of a salsa dance training on balance and strength performance in older adults. Gerontology. 2012;58(4):305-12. 
18. Sofianidis G, Hatzitaki V, Douka S, Grouios G. Effect of a 10-week traditional dance program on static and dynamic balance control in elderly adults. J Aging Phys Act. 2009;17:167-80.

19. Garber CE, Blissmer B, Deschenes MR, Franklin BA, Lamonte MJ, Lee IM, et al. American College of Sports Medicine position stand. Quantity and Quality of Exercise for Developing and Maintaining Cardiorespiratory, Musculoskeletal, and Neuromotor Fitness in Apparently Healthy Adults: guidance for prescribing exercise. Med Sci Sports Exerc. 2011;43(7):1334-59.

20. Borg G. Psychophysical bases of perceived exertion. Med Sci Sports Exerc. 1982;14(5):377-81.

21. Jones CJ, Rikli RE. Measuring functional. J Act Aging. 2002;24-30.

22. Rikli RE, Jones CJ. Senior Fitness Test Manual. Human Kinetics; 2012. 202 p.

23. Rikli RE, Jones JC. Development and Validation of a Functional Fitness Test for Community-Residing Older Adults. J Aging Phys Act. 1999;7:129-61.

24. Rinne MB, Pasanen ME, Miilunpalo SI, Oja P. Test-retest reproducibility and inter-rater reliability of a motor skill test battery for adults. Int J Sports Med. 2001;22:192-200.

25. Martínez EJ. Aplicación de la prueba de rotación de hombros con bastón, sit and reach y flexión profunda del cuerpo. Resultados y análisis estadístico en Educación Secundaria. Rev Int Med Cienc Act Física El Deporte. 2003;3(11):149-72.

26. Yung PS. Effects of weight bearing and non-weight bearing exercises on bone properties using calcaneal quantitative ultrasound. $\mathrm{Br} J$ Sports Med. 2005;39(8):547-51.

27. Nordström A, Tervo T, Högström M. The Effect of Physical Activity on Bone Accrual, Osteoporosis and Fracture Prevention. Open Bone J. 2011;(3):11-21.

28. Gourlan MJ, Trouilloud DO, Sarrazin PG. Interventions promoting physical activity among obese populations: a meta-analysis considering global effect, long-term maintenance, physical activity indicators and dose characteristics. Obes Rev. 2011;12(7):e633-45.

29. Eyigor S, Karapolat H, Durmaz B, Ibisoglu U, Cakir S. A randomized controlled trial of Turkish folklore dance on the physical performance, balance, depression and quality of life in older women. Arch Gerontol Geriatr. 2009;48(1):84-8.

30. Federici A, Bellagamba $S$, Rocchi MB. Does dance-based training improve balance in adult and young old subjects? A pilot randomized controlled trial. Aging Clin Exp Res. 2005;17(5):385-9.

31. 31. Dewhurst S, Nelson N, Dougall PK, Bampouras TM. Scottish Country Dance: Benefits to Functional Ability in Older Women. J Aging Phys Act. 2014;22(1):146-53.

32. Hackney ME, Earhart GM. Effects of Dance on Gait and Balance in Parkinson Disease: A Comparison of Partnered and Nonpartnered Dance Movement. Neurorehabil Neural Repair. 2010;24(4):384-92.

33. McKinley P, Jacobson A, Leroux A, Bednarczyk V, Rossignol M, Fung J. Effect of a community-based Argentine tango dance program on functional balance and confidence in older adults. J Aging Phys Act. 2008;16(4):435-53.

34. Oral A, Tarakçi D, Disçi R. Calcaneal quantitative ultrasound measurements in young male and female professional dancers. J Strength Cond Res. 2006;20(3):572-8.

35. Seco J, Abecia LC, Echevarría E, Barbero I, Torres-Unda J, Rodriguez V, et al. A long-term physical activity training program increases strength and flexibility, and improves balance in older adults. Rehabil Nurs. 2013;38(1):37-47.

\section{Referencias totales citadas: 35}

Referencias citadas correspondientes a la Rev Ib CC Act Fis Dep: 0 